\title{
Transport Relaxation Time of Spin-Polarized Electrons
}

\author{
A. PAJA* AND B.J. SPISAK \\ Faculty of Physics and Nuclear Techniques \\ AGH-University of Science and Technology \\ al. Mickiewicza 30, 30-059 Kraków, Poland
}

(Received December 1, 2003; revised version May 17, 2004)

We study the mechanism of spin relaxation in 3D disordered metallic systems due to the spin-orbit scattering on charged impurities. The transport relaxation time for spin-polarized conduction electrons is calculated analytically in the presented model, where the screened Coulomb potential is used for the description of impurities.

PACS numbers: 72.10.-d, 72.10.Fk, 72.15.Lh, 72.25.Ba, 72.25.Rb

\section{Introduction}

The transport of polarized electrons through $n$-dimensional systems $(n=1$, 2,3 ) is one of the most important problems in modern electronics (spintronics), where spin of conduction electrons plays a crucial role [1]. Particularly important interaction for spintronics is the spin-orbit (SO) interaction which causes the rotation of electron spin and gives rise to the spin relaxation phenomena, which are very important for industrial applications.

The spin-orbit interaction results from the coupling of electron spin with the static electric field. If a charged impurity is the source of such electric field then the SO interaction is called the Elliott-Yafet spin scattering mechanism [2]. This type of SO scattering plays a significant role in coherent transport in disordered systems, where it can dramatically change the properties of the system in the case of weak localization [3].

The transport of unpolarized electrons in 3D disordered metallic systems was treated in our previous paper [4]. Grimaldi [5] calculated the transport properties of $2 \mathrm{D}$ electron gas polarized in-plane by a magnetic field.

*corresponding author; e-mail: paja@uci.agh.edu.pl 
The purpose of this paper is to consider polarized electrons in 3D disordered system, which interact with rarely distributed metal impurities or other artificial structures. We do not consider here the problem of generation of the spin polarization, but we assume that polarized electrons are injected into the metallic system or heavily doped semiconductor. We neglect the interaction of electrons with positively charged matrix which is equivalent to the jellium model. Our description involves the spin-orbit interaction as an additional scattering mechanism. Using the presented model, we reckon the transport relaxation time in this case.

The paper is organized as follows. In Sec. 2 we give the theoretical background of presented model and point out its limitations. In Sec. 3 we calculate the transport relaxation time and in Sec. 4 we present numerical results and their discussion.

\section{Theoretical model}

We consider the spin dependent scattering of the conduction electrons from the impurity described by the spherically symmetric atomic potential $u_{\mathrm{a}}(r)$. The degree of disorder is low, and the Ioffe-Regel criterion [6] is assumed to be valid. It means that electrons can be treated as free particles propagating as plane waves between collisions $[6,7]$.

The nonmagnetic impurity introduced into the system produces the perturbation of conduction electrons in the following form:

$$
U(r)=u_{\mathrm{a}}(r)+a_{\mathrm{SO}} \widehat{\sigma} \cdot\left[\nabla u_{\mathrm{a}}(r) \times \widehat{\boldsymbol{p}}\right],
$$

where $\widehat{\boldsymbol{p}}$ is the momentum operator, $\widehat{\boldsymbol{\sigma}}$ denotes the Pauli spin matrices, $a_{\mathrm{SO}}=$ $\hbar /(2 m c)^{2}, \hbar$ and $c$ have their usual meaning. The first term in Eq. (1) represents the ordinary scattering and it does not affect the spin variable, and the second one represents the spin-orbit scattering.

The problem of the electron scattering by the potential can be described by the Lippman-Schwinger (LS) equation [8], which in this case has the following form:

$$
\boldsymbol{\Psi}(\boldsymbol{r})=\boldsymbol{\Phi}(\boldsymbol{r})-\frac{m^{*}}{2 \pi \hbar^{2}} \int \mathrm{d}^{3} r^{\prime} \frac{\exp \left(\mathrm{i} k\left|\boldsymbol{r}-\boldsymbol{r}^{\prime}\right|\right)}{\left|\boldsymbol{r}-\boldsymbol{r}^{\prime}\right|} U\left(r^{\prime}\right) \boldsymbol{\Psi}\left(\boldsymbol{r}^{\prime}\right),
$$

where $\boldsymbol{\Psi}(\boldsymbol{r})$ and $\boldsymbol{\Phi}(\boldsymbol{r})$ are spinors.

We use the effective mass $m^{*}$ instead of the reduced mass as it was in the original LS equation. This is a standard approximation at this level because we consider the scattering of the electron from the ion which is in a fixed position. On the second hand this allows us to include some specific properties of the system. The finding of the exact solution of Eq. (2) is impossible in general, however making some assumptions we can use one of the standard method of solving this type of integral equation, namely the Born approximation [8]. Using this method we can find the scattering amplitude $\widehat{\boldsymbol{F}}(\theta)$ which is directly related to the differential 
cross-section by the formula

$$
\frac{\mathrm{d} \sigma}{\mathrm{d} \Omega}=\left|\left\langle\boldsymbol{k}^{\prime}, s^{\prime}|\widehat{\boldsymbol{F}}(\theta)| \boldsymbol{k}, s\right\rangle\right|^{2},
$$

where $\widehat{\boldsymbol{F}}(\theta)$ is a $2 \times 2$ matrix in spin space, and the indices $s, s^{\prime}=\uparrow, \downarrow$ (referred to $z$-components of the electron spin). The standard interpretation of the elements of this matrix can be based on the well-known literature [9]. Its diagonal elements represent the scattering without spin-flip, and the off-diagonal ones describe the spin-flip processes during the scattering. The scattering amplitude can be decomposed into three parts: two diagonal ones and one off-diagonal as follows:

$$
\widehat{\boldsymbol{F}}(\theta)=\widehat{\boldsymbol{F}}_{0}(\theta)+\widehat{\boldsymbol{F}}_{\mathrm{nsf}}(\theta)+\widehat{\boldsymbol{F}}_{\mathrm{sf}}(\theta),
$$

where $\widehat{\boldsymbol{F}}_{0}(\theta)$ is the normal scattering amplitude, $\widehat{\boldsymbol{F}}_{\text {nsf }}(\theta)$ is a non-spin-flip one, and $\widehat{\boldsymbol{F}}_{\text {sf }}(\theta)$ is a spin-flip one.

In this case, the asymptotic form of the total wave function $\boldsymbol{\Psi}(\boldsymbol{r})$ can be written as

$$
\boldsymbol{\Psi}(\boldsymbol{r})=\Phi(\boldsymbol{r})+\widehat{\boldsymbol{F}}_{0}(\theta) \frac{\mathrm{e}^{\mathrm{i} k r}}{r}+\widehat{\boldsymbol{F}}_{\mathrm{nsf}}(\theta) \frac{\mathrm{e}^{\mathrm{i} k r}}{r}+\widehat{\boldsymbol{F}}_{\mathrm{sf}}(\theta) \frac{\mathrm{e}^{\mathrm{i} k r}}{r} .
$$

We can estimate the scattering amplitude using the lowest Born approximation. The use of this approximation means that the scattering amplitude is proportional to the matrix element of the perturbation potential $U(r)$ taken between the plane waves representing the conduction electron before and after the scattering. These plane waves are normalized in the way typical of the theory of scattering: one particle per unit volume. The matrix elements can be denoted as $M_{s^{\prime} s}\left(\boldsymbol{k}^{\prime}, \boldsymbol{k}\right)=$ $\left\langle\boldsymbol{k}^{\prime}, s|U(r)| \boldsymbol{k}, s\right\rangle$. The matrix element for the ordinary potential is given by

$$
M_{s^{\prime} s}^{0}\left(\boldsymbol{k}^{\prime}, \boldsymbol{k}\right)=\tilde{u}_{\mathrm{a}}(\boldsymbol{q}) \delta_{s^{\prime} s},
$$

where $\tilde{u}_{\mathrm{a}}(\boldsymbol{q})$ is the Fourier transform of the atomic potential, $\hbar \boldsymbol{q}$ is the momentum transfer.

But the matrix element for the spin-orbit part has the form

$$
M_{s^{\prime} s}^{\mathrm{SO}}\left(\boldsymbol{k}^{\prime}, \boldsymbol{k}\right)=a_{\mathrm{SO}} \int \mathrm{d}^{3} r w(r) \mathrm{e}^{-\mathrm{i} \boldsymbol{k}^{\prime} \cdot \boldsymbol{r}} \boldsymbol{\sigma}_{s^{\prime} s} \cdot \widehat{\boldsymbol{L}} \mathrm{e}^{\mathrm{i} \boldsymbol{k} \cdot \boldsymbol{r}}
$$

where $w(r)$ is a radial function defined by

$$
w(r)=\frac{1}{r} \frac{\mathrm{d} u_{\mathrm{a}}(r)}{\mathrm{d} r}
$$

and $\widehat{\boldsymbol{L}}$ is the orbital angular momentum operator.

For the particular spin states, e.g. $s^{\prime}=\downarrow$ and $s=\uparrow$, this result can be rewritten in the following form:

$$
M_{\downarrow \uparrow}^{\mathrm{SO}}\left(\boldsymbol{k}^{\prime}, \boldsymbol{k}\right)=a_{\mathrm{SO}} \int \mathrm{d}^{3} r w(r) \mathrm{e}^{-\mathrm{i} \boldsymbol{k}^{\prime} \cdot \boldsymbol{r}} \widehat{\boldsymbol{L}}_{+} \mathrm{e}^{\mathrm{i} \boldsymbol{k} \cdot \boldsymbol{r}},
$$

where we introduced the ladder operator for the orbital angular momentum. In 
analogous way the $\widehat{\boldsymbol{L}}_{-}$operator corresponds to the $M_{\uparrow \downarrow}^{\mathrm{SO}}\left(\boldsymbol{k}^{\prime}, \boldsymbol{k}\right)$. This form of $M_{\downarrow \uparrow}^{\mathrm{SO}}\left(\boldsymbol{k}^{\prime}, \boldsymbol{k}\right)$ shows that the flip of the spin must be compensated by the change of the orbital angular momentum. It can be interpreted as the conservation of angular momentum during the scattering of electrons off the spherically symmetric potentials.

The total differential cross-section for 'up'-polarized electrons in the first Born approximation has the following form:

$$
\frac{\mathrm{d} \sigma}{\mathrm{d} \Omega}=\frac{\mathrm{d} \sigma_{0}}{\mathrm{~d} \Omega}+\frac{\mathrm{d} \sigma_{\uparrow \uparrow}}{\mathrm{d} \Omega}+\frac{\mathrm{d} \sigma_{\downarrow \uparrow}}{\mathrm{d} \Omega}
$$

where the first term on the right hand side is given by the formula

$$
\frac{\mathrm{d} \sigma_{0}}{\mathrm{~d} \Omega}=\left(\frac{m^{*}}{2 \pi \hbar^{2}}\right)^{2}\left|M_{\uparrow \uparrow}^{0}\left(\boldsymbol{k}^{\prime}, \boldsymbol{k}\right)\right|^{2},
$$

the second one is given by

$$
\frac{\mathrm{d} \sigma_{\uparrow \uparrow}}{d \Omega}=\left(\frac{m^{*}}{2 \pi \hbar^{2}}\right)^{2}\left|M_{\uparrow \uparrow}^{\mathrm{SO}}\left(\boldsymbol{k}^{\prime}, \boldsymbol{k}\right)\right|^{2},
$$

and the third one by

$$
\frac{\mathrm{d} \sigma_{\downarrow \uparrow}}{\mathrm{d} \Omega}=\left(\frac{m^{*}}{2 \pi \hbar^{2}}\right)^{2}\left|M_{\downarrow \uparrow}^{\mathrm{SO}}\left(\boldsymbol{k}^{\prime}, \boldsymbol{k}\right)\right|^{2} .
$$

\section{Transport relaxation time}

Let us assume the Coulomb screened potential as a more realistic approximation for the scattering impurities in the form

$$
u_{\mathrm{a}}(r)=u_{0} \frac{\mathrm{e}^{-\lambda r}}{r},
$$

where $u_{0}$ is a strength of the potential, and $\lambda$ is the inverse of screening length of the bare Coulomb potential. It reduces the range of influence of the impurities. If the concentration of the impurities is low, then they can be considered as independent. It means that we can reckon the scattering rate for a single impurity.

However the more realistic model of disorder should include some correlations between impurities. One of the simple realizations of this task is the introduction of the structure factor [10] to the scattering rate as it was presented in Ref. [4]. In the considered case, we assume the delta-like structure factor and therefore the impurities are treated as independent ones.

It is not difficult to calculate the matrix element (6) for the Coulomb screened potential and the adequate differential cross-section has the form

$$
\frac{\mathrm{d} \sigma_{0}}{\mathrm{~d} \Omega}=\left(\frac{2 m^{*} u_{0}}{\hbar^{2}}\right)^{2} \frac{1}{\left(\lambda^{2}+q^{2}\right)^{2}} .
$$


Calculation of the matrix elements for the spin-orbit part $M_{s^{\prime} s}^{\mathrm{SO}}\left(\boldsymbol{k}^{\prime}, \boldsymbol{k}\right)$ gives rise to integrals proportional to $\left(\boldsymbol{k} \times \boldsymbol{k}^{\prime}\right)_{z}$ for non-spin-flip events and to $\left(\boldsymbol{k} \times \boldsymbol{k}^{\prime}\right)_{x}$ $+\mathrm{i}\left(\boldsymbol{k} \times \boldsymbol{k}^{\prime}\right)_{y}$ for spin-flip ones. Let us assume the current of electrons polarized along the $z$-axis and denote the angle between this axis and the vector $\boldsymbol{k} \times \boldsymbol{k}^{\prime}$ normal to the scattering plane by $\xi$. Then the differential cross-sections for these two kinds of scattering differ only by the factor dependent on $\xi$ :

$$
\frac{\mathrm{d} \sigma_{\mathrm{nsf}}}{\mathrm{d} \Omega}=\left(\frac{b_{\mathrm{SO}} m^{*} u_{0}}{\hbar^{2}}\right)^{2} \frac{\lambda^{-4} q^{4}}{\left(1+\lambda^{-2} q^{2}\right)^{2}} \cot ^{2}\left(\frac{\theta}{2}\right) \cos ^{2} \xi
$$

and

$$
\frac{\mathrm{d} \sigma_{\mathrm{sf}}}{\mathrm{d} \Omega}=\left(\frac{b_{\mathrm{SO}} m^{*} u_{0}}{\hbar^{2}}\right)^{2} \frac{\lambda^{-4} q^{4}}{\left(1+\lambda^{-2} q^{2}\right)^{2}} \cot ^{2}\left(\frac{\theta}{2}\right) \sin ^{2} \xi
$$

where $b_{\mathrm{SO}}=\hbar a_{\mathrm{SO}}$.

It is interesting to consider two special cases. If the axis of quantization is chosen parallel to $\boldsymbol{k}$ and the incident electrons are fully polarized then $\xi=\pi / 2$ and all spins are reversed due to scattering. The result is identical as presented in Grimaldi work [5] but he considered 2D problem and we solved 3D one here. For other orientations of the $z$-axis we can observe both reversed and non-reversed spins. If the $z$-axis is perpendicular to $\boldsymbol{k}$ the angle $\xi$ can take any value and we have to average both cross-sections over the whole range, i.e. $2 \pi$. It results in equal probability for both spin-flip and non-spin-flip scatterings.

The description of electron transport phenomena in solids can be based on the semiclassical Boltzmann equation, where the collision term is evaluated in the relaxation time approximation [11]. The transport relaxation time is given by the formula

$$
\tau_{\mathrm{tr}}^{-1}=2 \pi \frac{v_{\mathrm{F}}}{V} \int_{0}^{\pi} \mathrm{d} \theta \sin \theta \frac{\mathrm{d} \sigma}{\mathrm{d} \Omega}(1-\cos \theta),
$$

where $v_{\mathrm{F}}$ is the Fermi velocity, $V$ is the volume of the system, $\theta$ is the scattering angle, and differential cross-section is given by the formula (9) with the definitions $(10,11,12)$.

The reckoning of the transport relaxation time is based on integral (17) and gives three contributions to this formula, namely:

for the ordinary scattering

$$
\left(\tau_{\mathrm{tr}}^{-1}\right)_{0}=2 \pi \frac{v_{\mathrm{F}}}{V}\left(\frac{2 m^{*} u_{0}}{\lambda^{2} \hbar^{2}}\right)^{2} f(x),
$$

for non-spin-flip scattering

$$
\left(\tau_{\mathrm{tr}}^{-1}\right)_{\mathrm{nsf}}=\frac{\pi}{2} \frac{v_{\mathrm{F}}}{V}\left(\frac{b_{\mathrm{SO}} m^{*} u_{0}}{\hbar^{2}}\right)^{2} g(x) \cos ^{2} \xi,
$$

and for spin-flip scattering 


$$
\left(\tau_{\mathrm{tr}}^{-1}\right)_{\mathrm{sf}}=\frac{\pi}{2} \frac{v_{\mathrm{F}}}{V}\left(\frac{b_{\mathrm{SO}} m^{*} u_{0}}{\hbar^{2}}\right)^{2} g(x) \sin ^{2} \xi,
$$

where $x=k / \lambda$, and functions $f(x)$ and $g(x)$ are defined as follows:

$$
f(x)=\frac{2 x^{2}}{\left(1+4 x^{2}\right)}+\frac{1}{4} \frac{\ln \left(1+4 x^{2}\right)}{x^{4}}
$$

and

$$
\begin{aligned}
g(x) & =\frac{1}{2}+\frac{1}{4 x^{2}}\left[3-2 \ln \left(1+4 x^{2}\right)-\frac{1}{1+4 x^{2}}\right] \\
& +\frac{1}{16 x^{4}}\left[1-3 \ln \left(1+4 x^{2}\right)-\frac{1}{1+4 x^{2}}\right] .
\end{aligned}
$$

Our approach can be compared with the one which uses the self-consistent Born approximation for the self-energy [5]. This approximation exploits the Green function methods. The averaged Green function $\langle G(\boldsymbol{k}, E)\rangle$ can be expressed by the free electron Green function $G_{0}(\boldsymbol{k}, E)$ and a self-energy $\Sigma(\boldsymbol{k}, E)$, as follows:

$$
\langle G(\boldsymbol{k}, E)\rangle^{-1}=G_{0}^{-1}(\boldsymbol{k}, E)-\Sigma(\boldsymbol{k}, E) .
$$

On the other hand, the self-energy can be calculated from the following formula:

$$
\Sigma(\boldsymbol{k}, E)=\frac{N}{V} \sum_{\boldsymbol{k}^{\prime}} U\left(\boldsymbol{k}, \boldsymbol{k}^{\prime}\right)\left\langle G\left(\boldsymbol{k}^{\prime}, E\right)\right\rangle U\left(\boldsymbol{k}^{\prime}, \boldsymbol{k}\right) .
$$

Inverse relaxation time $\tau^{-1}$ is related to $\Sigma(\boldsymbol{k}, E)$ by the following formula [12]:

$$
|\Im\{\Sigma(\boldsymbol{k})\}|=\hbar \tau^{-1} .
$$

The set of equations (23) and (24) determines the self-consistent procedure for self-energy. Calculation of the self-energy in the second order of the perturbation theory is equivalent to our result.

\section{Numerical results and discussion}

The spin-orbit contribution to the resistivity can be estimated from the ratio $\varepsilon=\left(\tau_{\mathrm{tr}}^{-1}\right)_{\mathrm{SO}} /\left(\tau_{\mathrm{tr}}^{-1}\right)_{0}$, where $\left(\tau_{\mathrm{tr}}^{-1}\right)_{\mathrm{SO}}=\left(\tau_{\mathrm{tr}}^{-1}\right)_{\mathrm{sf}}+\left(\tau_{\mathrm{tr}}^{-1}\right)_{\mathrm{nsf}}$. It is easily seen that

$$
\varepsilon=\frac{1}{16} b_{\mathrm{SO}}^{2} \lambda^{4} \frac{g(x)}{f(x)} .
$$

The inverse of screening length $\lambda$ is calculated within the Thomas-Fermi model [7]. Then the parameter $x$ can be expressed as

$$
x \approx 1.227\left(\frac{r_{s}}{a_{0}}\right)^{-1 / 2},
$$

where the values of dimensionless parameter $r_{s} / a_{0}$ for some metals are given in Ref. [7]. 
The numerical values of $x$ are in the range between 0.5 and 0.68 (for alkali metals). The ratio $g(x) / f(x)$ is of the order of unity in this range (see Fig. 1).

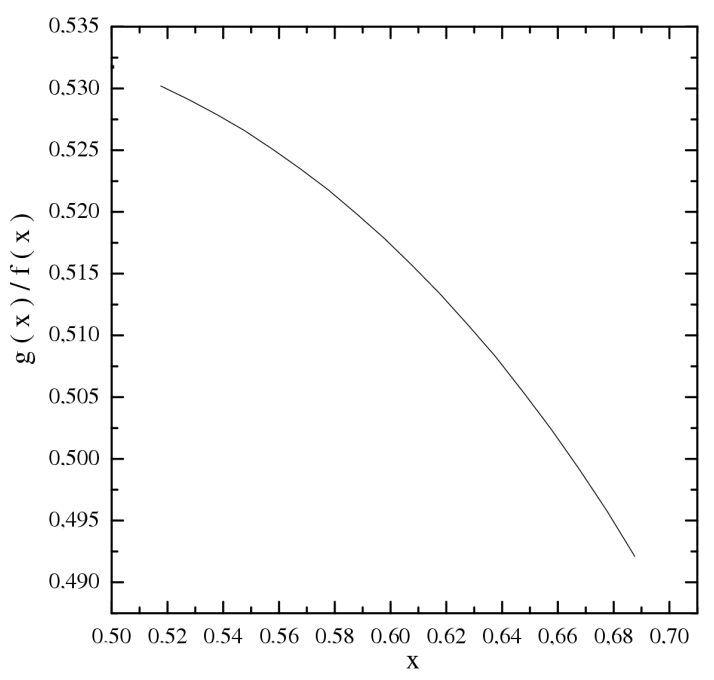

Fig. 1. The ratio of $g(x) / f(x)$ as a function of the dimensionless parameter $x$.

The SO coupling constant, $b_{\mathrm{SO}}$, equals to $3.43 \times 10^{-26} \mathrm{~m}^{-1}$ whereas $\lambda$ is of the order of $10^{10} \mathrm{~m}^{-1}$ which gives $\varepsilon \approx 10^{-12}$. Experimentally measured $\varepsilon$ is rather closer to the value of $10^{-4}$ (Ref. [13]) though other authors [14] give more dispersed values of $\varepsilon$ : from $10^{-7}$ to $10^{-1}$. We have to remember that the value of $\lambda$ has been estimated on the ground of the free electron theory. The description of incident and scattered particles by plane waves is valid only asymptotically, that is at long distance from the scattering centre. Replacing $\lambda$ by $\lambda_{\text {eff }} \gg \lambda$ would give better agreement with experiment. Physically, it means a much smaller range of potential. Many authors model a short range of potentials in the theory of metals by $\delta$-functions. Assuming $\lambda_{\text {eff }}$, which corresponds to short-range but not $\delta$-like potential, would be a more realistic description. In any case the ratio $\varepsilon$ is rather small. It means that the SO scattering gives a negligible contribution to the total resistivity if we calculate it classically by using Matthiesen's rule.

In our previous paper [4] we used the structure factor which took into account the correlations between positions of ions according to the random close packed hard spheres model. In this work we assume randomly distributed isolated metal impurities which therefore are totally uncorrelated (i.e. the structure factor is constant in this case).

One of the most important results of this work is a finding that a beam of electrons fully polarized in the direction parallel to the current density comes out totally polarized in the reverse direction. In the other special case, when electrons are fully polarized in the direction perpendicular to the current they come out 
totally depolarized. These conclusions may be important for spintronics where the spin of electron is a carrier of information on equal footing as the charge.

However, if we include the multiple scattering processes which give rise to the weak localization effects [15] in disordered systems, then the influence of the SO interaction on the transport phenomena is much more stronger, as it is well known $[16,17]$.

\section{Acknowledgments}

The authors thank Prof. J. Barnaś of the A. Mickiewicz University for helpful discussions.

\section{References}

[1] G. Prinz, Phys. Today 48, 58 (1995).

[2] R.J. Elliott, Phys. Rev. 96, 266 (1954).

[3] G. Bergmann, Phys. Rev. Lett. 48, 1046 (1982).

[4] B. Spisak, A. Paja, Acta Phys. Pol. A 96, 751 (1999).

[5] C. Grimaldi, J. Phys. C 12, 1329 (2000).

[6] B.L. Altshuler, A.G. Aronov, in: Electron-Electron Interactions in Disordered Systems Eds. A.L. Efros, M. Pollak, North-Holland, Amsterdam 1985, p. 1.

[7] N.W. Ashcroft, N.D. Mermin, Solid State Physics, Holt, Rinehart and Winston, New York 1976.

[8] F. Byron, R. Fuller, Mathematics of Classical and Quantum Physics, Vol. 2, PWN, Warszawa 1975 (in Polish).

[9] E. Merzbacher, Quantum Mechanics, Wiley, New York 1998.

[10] J.M. Ziman, Philos. Mag. 6, 1013 (1961).

[11] J.M. Ziman, Electrons and Phonons, Clarendon Press, Oxford 2001.

[12] L. Schwartz, A. Paja, Phys. Status Solidi B 118, 101 (1983).

[13] A. Sahnoune, J.O. Ström-Olsen, H.E. Fischer, Phys. Rev. B 46, 10035 (1992).

[14] R. Meservey, P.M. Tedrow, Phys. Rev. Lett. 41, 805 (1978).

[15] G. Bergmann, Phys. Rep. 107, 1 (1984).

[16] S. Hikami, A.I. Larkin, Y. Nagaoka, Prog. Theor. Phys. 63, 707 (1980).

[17] H. Fukuyama, K. Hoshino, J. Phys. Soc. Jpn. 50, 2131 (1981). 